Fluctuation and Noise Letters

Vol. 7, No. 2 (2007) L111-L133

(c) World Scientific Publishing Company

World Scientific

www.worldscientific.com

\title{
PHASE DYNAMICS AND KINETICS OF THIN LUBRICANT FILM DRIVEN BY CORRELATED TEMPERATURE FLUCTUATIONS
}

\author{
ALEXEI V. KHOMENKO, IAKOV A. LYASHENKO \\ Sumy State University, Rimskii-Korsakov St. 2, 40007 Sumy, Ukraine \\ khom@phe.sumdu.edu.ua,nabla@cable-tv.sumy.ua \\ Received 17 November 2006 \\ Revised 13 April 2007 \\ Accepted 13 April 2007 \\ Communicated by Andras Der
}

\begin{abstract}
The melting of an ultrathin lubricant film is studied at friction between atomically flat surfaces. We take into account fluctuations of lubricant temperature, which are defined by the Ornstein-Uhlenbeck process. Phase diagrams and portraits are calculated for second- and first-order transitions (the melting of an amorphous and that of a crystalline lubricants, respectively). It is shown that, in the former case, a stick-slip friction domain, separating the regions of dry and sliding friction, appears. In the latter case, three domains of stick-slip friction arise, which are characterized by the transitions between dry, metastable and stable sliding friction. The increase in the correlation time of lubricant temperature fluctuations leads to increasing in the rubbing-surface temperature needed for realization of sliding friction. The stationary states, corresponding to dry, stable and metastable sliding friction, are reached as a result of damped oscillations.
\end{abstract}

Keywords: Viscoelastic medium; melting; stick-slip friction; white noise; OrnsteinUhlenbeck process.

\section{Introduction}

Sliding friction remains a subject of extensive research due to wide technological application of smooth solid surfaces, which are separated by a thin film of lubricant [1-3]. It was found experimentally that during the process of friction a liquid film became progressively thinner, at first its physical properties changed gradually (quantitatively), and then changes acquired the sharp (qualitative) character. The qualitative changes include the non-Newtonian mechanism of flow and replacement of the ordinary melting by glass transition however, the lubricant film continues to behave like a liquid. In tribology such behaviour is called "mixed lubrication", which represents an intermediate mode of friction, that is characterized by transition from the bulk to boundary properties of a lubricating material. 
We describe a boundary mode of friction, which is observed in the case of an ultrathin (less than four molecular diameters thick) lubricant film between smooth or rough surfaces, high loads, and low shear rates. It is characterized by the following changes of static (equilibrium) and dynamic properties of lubricant (simple unstructured Newtonian liquid) [3]:

- non-fluid-like (non-Newtonian) properties: transition between liquid and solid phases, appearance of new liquid-crystalline states, epitaxially induced longrange ordering;

- tribological properties: absence of flow until yield point or critical shear stress reached, solid-like behaviour of lubricant, that is characterized by defect diffusion and dislocation motion, shear and jump-like melting, boundary lubrication.

Experiments were carried out with mica, silica, metal oxide, and surfactant monolayer surfaces with organic liquids and aqueous solutions in between. It has been shown that there were transformations between different types of dynamic phases during sliding [3]. These transformations show up as intermittent (stick-slip) friction [4-7], which is characterized by periodic transitions between two or more dynamic states during the stationary sliding. The stick-slip friction is the major reason for destruction and wear of rubbing parts. Thus, molecularly thin lubricant films experience one or more types of transitions, which give rise to different modes of stick-slip motion.

In particular, experimental investigation of rheological properties of the lubricant film has elucidated the nature of such anomalies in boundary friction [3]. Besides, to this end, theoretical presentation of the lubricating material was used as a viscoelastic matter with non-zero heat conductivity. So, the phenomenology of the ultrathin lubricant film observed in experiment is successfully described in [7] on the basis of the viscoelastic matter approximation and Ginzburg-Landau equation, in which the order parameter defines shear melting and freezing. A dynamic phase diagram is calculated defining the domains of sliding, stick-slip, and dry friction in the film temperature - film thickness coordinates.

Extending work [7], in terms of the Lorentz model of viscoelastic matter [8] the transition of the ultrathin lubricant film from a solid-like to a liquid-like state is shown to occur due to both thermodynamic and shear melting. A cooperative description of these processes is given under the assumption that they result from the self-organization of shear stress and stain, and the lubricant temperature. The notion of additive noise of these quantities is introduced and phase diagrams are constructed, where fluctuation intensity and rubbing-surface temperature define the domains of sliding, stick-slip, and dry friction [9]. In Ref. [10] the conditions under which the stick-slip friction regime corresponds to the intermittency mode inherent in self-organized criticality phenomenon are found. Effect of different types of temperature dependence of viscosity on the melting temperature of lubricant is taken into account [11].

However, in spite of the fact that temperature, as a rule, determines the lubricant state, the issue of how correlated temperature fluctuations influence the process of friction remains open. In this paper it is shown that internal fluctuations of temperature, which are described by the Ornstein-Uhlenbeck process, complicate 
the dynamic phase diagram. The shear stress distribution, phase diagrams and portraits are calculated for the second- and first-order transitions (melting of the amorphous and crystalline lubricant; Sections 4 and 5 , respectively). The different stick-slip friction modes are predicted to occur. The effect of fluctuation correlation time on the phase diagram is described. Kinetic modes of boundary friction are discussed using the phase plane method. Self-similar phase dynamics and kinetics of the lubricant film are investigated.

\section{Basic Equations}

In the previous work [8] we treated a viscoelastic medium with a non-zero thermal conductivity in rheological terms and derived a system of kinetic equations describing the mutually coordinated evolution of shear stresses $\sigma$ and strain $\varepsilon$, and temperature $T$ in an ultrathin lubricant film during friction between atomically flat mica surfaces. Let us write these equations using the measure units

$$
\sigma_{s}=\left(\frac{\rho c_{v} \eta_{0} T_{c}}{\tau_{T}}\right)^{1 / 2}, \quad \varepsilon_{s}=\frac{\sigma_{s}}{G_{0}} \equiv\left(\frac{\tau_{\varepsilon}}{\tau_{T}}\right)^{1 / 2}\left(\frac{\rho c_{v} T_{c} \tau_{\varepsilon}}{\eta_{0}}\right)^{1 / 2}, \quad T_{c}
$$

for variables $\sigma, \varepsilon, T$, respectively, where $\rho$ is the lubricant density, $c_{v}$ is the specific heat capacity, $T_{c}$ is the critical temperature, $\eta_{0} \equiv \eta\left(T=2 T_{c}\right)$ is the characteristic value of shear viscosity $\eta, \tau_{T} \equiv \rho l^{2} c_{v} / \kappa$ and $l$ are the time and scale of heat conduction, $\kappa$ is the thermal conductivity, $\tau_{\varepsilon}$ is the relaxation time of matter strain, $G_{0} \equiv \eta_{0} / \tau_{\varepsilon}:$

$$
\begin{aligned}
& \tau_{\sigma} \dot{\sigma}=-\sigma+g \varepsilon, \\
& \tau_{\varepsilon} \dot{\varepsilon}=-\varepsilon+(T-1) \sigma, \\
& \tau_{T} \dot{T}=\left(T_{e}-T\right)-\sigma \varepsilon+\sigma^{2}+\lambda(t) .
\end{aligned}
$$

Here the stress relaxation time $\tau_{\sigma}$, the temperature $T_{e}$ of atomically flat mica friction surfaces, and the constant $g=G / G_{0}$ are introduced, where $G$ is the lubricant shear modulus. By substituting $\varepsilon / \tau_{\sigma}$ for $\partial \varepsilon / \partial t$, Eq. (2) reduces to a Maxwell-type equation for a viscoelastic matter, which is widely used in the theory of boundary friction [1]. The relaxation behaviour of a viscoelastic lubricant during friction is also described by the Kelvin-Voigt equation $(3)[8,12]$. It takes into account the dependence of the shear viscosity on the dimensionless temperature $\eta=\eta_{0} /(T-1)[13]$. Taken together, equations (2) and (3) represent a new rheological model. It is worth noting that the rheological properties of the lubricant film are studied experimentally, which allows construction of phase diagrams [3]. Equation (4) represents the heat conduction expression, which describes heat transfer from the friction surfaces to the layer of lubricant, the dissipative heating of the stress-induced viscous flow, and a heat source due to the reversible mechanocaloric effect. Equations (2) - (4) formally coincide with the Lorentz synergetic system [14,15], where the shear stress acts as the order parameter, the conjugate field is reduced to the shear strain, and the temperature is the control parameter. As it is known this system can be used for description of both thermodynamic phase and kinetic transitions.

The purpose of this work is to study the phase dynamics and kinetics of boundary friction by introducing stochastic source $\lambda(t)$ into Eq. (4). This source represents 
the Ornstein-Uhlenbeck process [16]

$$
\langle\lambda(t)\rangle=0, \quad\left\langle\lambda(t) \lambda\left(t^{\prime}\right)\right\rangle=\frac{I}{\tau_{\lambda}} \exp \left(-\frac{\left|t-t^{\prime}\right|}{\tau_{\lambda}}\right)
$$

with the correlation time $\tau_{\lambda}$ and intensity $I$. Here $\lambda$ and $I$ are measured in units of

$$
\lambda_{s}=T_{c}, \quad I_{s}=T_{c}^{2} .
$$

The Ornstein-Uhlenbeck process $\lambda(t)$ presents realization of correlated random walks. This implies that with increase in velocity of particle the probability of its collision with another particle increases. Thus, all particles go back to zero velocity much rapidly than larger its value. The process is the colored Gaussian noise and it can be obtained by solving the Ornstein-Uhlenbeck equation:

$$
\frac{d \lambda(t)}{d t}=-\frac{1}{\tau_{\lambda}} \lambda(t)-\frac{1}{\tau_{\lambda}} \xi(t),
$$

where $\xi(t)$ represents the Gaussian white noise with zero average value and intensity $I$ :

$$
\langle\xi(t)\rangle=0, \quad\left\langle\xi(t) \xi\left(t^{\prime}\right)\right\rangle=2 I \delta\left(t-t^{\prime}\right) .
$$

Thus, source $\lambda(t)$ is similar to the white noise $\xi(t)$ on large time intervals and to the brownian motion on small ones, and it has moments (5) in the stationary limit $\left(t, t^{\prime} \gg \tau_{\lambda}\right)$.

Multiplying (7) by $d t$, we obtain the stochastic differential equation:

$$
d \lambda(t)=-\frac{1}{\tau_{\lambda}} \lambda(t) d t-\frac{1}{\tau_{\lambda}} d W(t) .
$$

Here the Winner process

$$
d W(t)=W(t+d t)-W(t) \equiv \xi(t) d t
$$

has the properties of the white noise [16]:

$$
\langle d W(t)\rangle=0 ; \quad\left\langle(d W(t))^{2}\right\rangle=2 I d t .
$$

For numerical realization of $\lambda(t)$ we solve equation (9) using the Euler method. Taking into account the definition of discrete analogue of random force differential $d W(t) \equiv \sqrt{\Delta t} W_{n}$ the following iterated procedure is obtained:

$$
\lambda_{n+1}=\lambda_{n}\left(1-\frac{\Delta t}{\tau_{\lambda}}\right)-\frac{\sqrt{\Delta t}}{\tau_{\lambda}} W_{n} .
$$

The solving is made on the interval $t \in[0, T]$, and the time increment is defined as $\Delta t=T / N$ at the fixed number of iterations $N$. Random force $W_{n}$ has the following properties:

$$
\left\langle W_{n}\right\rangle=0, \quad\left\langle W_{n} W_{n^{\prime}}\right\rangle=0, \quad\left\langle W_{n}^{2}\right\rangle \rightarrow 2 I .
$$

Its adequate mathematical model is the Box-Muller one [17]:

$$
W_{n}=\mu \sqrt{-2 \ln r_{1}} \cos \left(2 \pi r_{2}\right), \quad r_{i} \in(0,1],
$$


where $\mu^{2}=2 I$ is the dispersion. Pseudo-random numbers $r_{1}$ and $r_{2}$ repeat themselves through a certain interval.

The equality $\xi(t)=d W / d t$ follows from (10). The Winner process is undifferentiated although it represents a continuous function, i.e., without dependence on the value $d t$ the indicated derivative is almost certainly equal to infinity. This fact agrees with the expression for the second moment (8) that tends to infinity at $t=t^{\prime}$. In real physical systems the infinite dispersion is not realized, therefore $\delta(0)$ is assumed to be equal to a fixed number. In this connection for presentation of the white noise we consider the random force (14) with properties (13), which coincide with $(8)$ at $\delta(0) \equiv 1$.

The realization of (14) is represented for different time scales in Fig. 1. The pattern of behaviour for both scales is similar, and the noise is uncorrelated here.

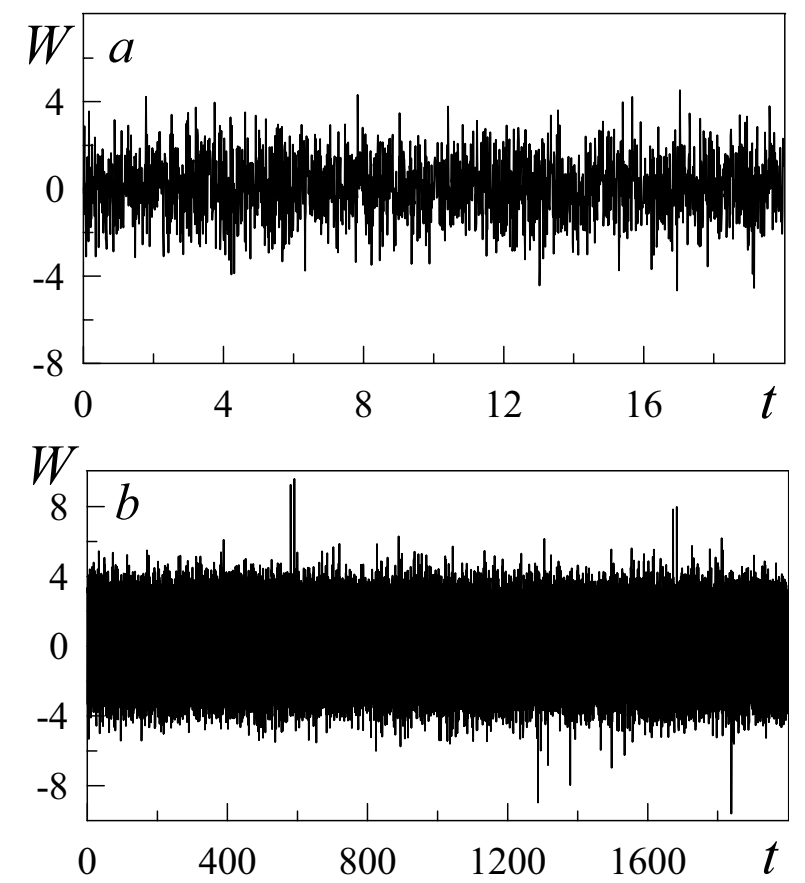

Fig. 1. White noise realization (14) at $I=1$. a: $N=2 \cdot 10^{3}, T=20, \Delta t=0.01$. b: $N=2 \cdot 10^{5}$, $T=2 \cdot 10^{3}, \Delta t=0.01$.

The Ornstein-Uhlenbeck process $\lambda(t)$ is presented for the same time intervals in Fig. 2. Apparently that it is similar to the brownian motion on short intervals (Fig. 2a), and here each subsequent value depends on a previous one, i.e., the noise is correlated. The correlation is lost on long intervals (Fig. 2b), the process becomes similar to the white noise whose realization is shown in Fig. 1. In contrast to the white noise the stationary Ornstein-Uhlenbeck process has finite dispersion, and it is often used as a model of a real noise signal.

Following [18], let us clarify the physical meaning of quantity $I$. The time correlation of the dimension temperature $T_{D}$ of lubricant is determined by the 

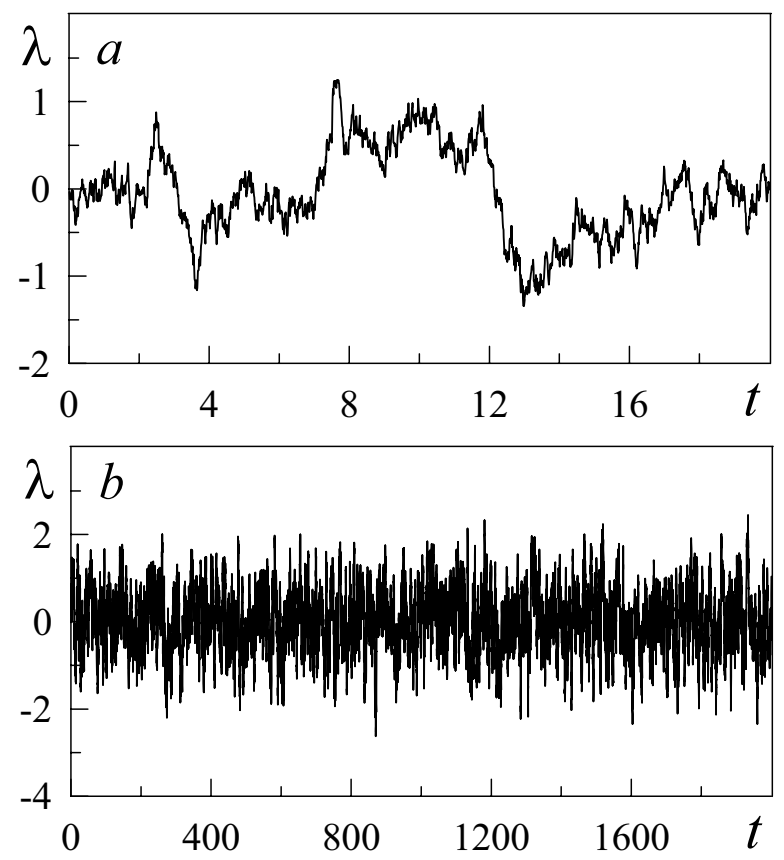

Fig. 2. Colored noise realization (12) at $I=1, \tau_{\lambda}=2.0$. a: $N=2 \cdot 10^{3}, T=20, \Delta t=0.01$. b: $N=2 \cdot 10^{5}, T=2 \cdot 10^{3}, \Delta t=0.01$.

average value of product

$$
\varphi(\tau)=\left\langle\Delta T_{D}(t) \Delta T_{D}(t+\tau)\right\rangle,
$$

where $\Delta T_{D}(t)$ is the difference between the current and the mean temperatures. Under the assumption that fluctuations $\Delta T_{D}$ are quasi-stationary, function $\varphi(\tau)$ accepts the form:

$$
\varphi(\tau)=\left\langle\left(\Delta T_{D}\right)^{2}\right\rangle \exp (-\zeta|\tau|)
$$

Here $1 / \zeta$ fixes the relaxation time to equilibrium. Supposing that the time instants in formulas (15) and (16) are connected by equality $t+\tau=t^{\prime}$, we get:

$$
\varphi\left(t^{\prime}-t\right)=\left\langle\Delta T_{D}(t) \Delta T_{D}\left(t^{\prime}\right)\right\rangle=\left\langle\left(\Delta T_{D}\right)^{2}\right\rangle \exp \left(-\zeta\left|t^{\prime}-t\right|\right) .
$$

Then, substitution of average square of temperature fluctuations $\left\langle\left(\Delta T_{D}\right)^{2}\right\rangle=T_{D}^{2} / c_{v}$ into expression (17) gives

$$
\left\langle\Delta T_{D}(t) \Delta T_{D}\left(t^{\prime}\right)\right\rangle=\frac{T_{D}^{2}}{c_{v}} \exp \left(-\zeta\left|t^{\prime}-t\right|\right) .
$$

Comparing this formula with the second equality in (5) and taking into account the measure units (6) and the last of (1) for dimensionless quantities $\lambda, T$, and $I$, we obtain:

$$
\lambda(t)=\Delta T(t), \quad \tau_{\lambda}=\frac{1}{\zeta}, \quad I=\frac{T^{2}}{\zeta c_{v}} .
$$


According to (19), noise intensity $I$ is defined by the temperature and the heat capacity $c_{v}$ of the lubricant. At first glance, it appears that there exists a one-to-one correspondence between $I$ and $T_{e}$ in the phase diagrams $T_{e}(I)$ shown in Figs. 4 and 7 and so a trajectory, instead of regions, must exist in the given coordinates. The motion along this curve describes the evolution of the system. However, this is not the case, since $T_{e}$ represents the thermostat temperature, which, varying arbitrarily, does not uniquely determine temperature $T$. We suppose that, due to the system's self-organization, a certain value of $T_{e}$ corresponds to a manifold values of $T$ and, in accordance with (19), to intensities $I$ at different time instants. Besides, the variation of $c_{v}$ leads to the change of $I$ in the course of time. Thus, this implies the existence of the phase diagram. It is also possible to change the intensity $I$ due to arbitrary choice of parameters $\zeta$ and $T_{c}$ characterizing a specific system.

However, in this paper, temperature fluctuations are meant in the wider sense. The fact is that thermal actions are, as a rule, caused by external stochastic sources. Therefore, noise can equally be treated in terms of fluctuations of $T$ or $T_{e}$. Such an interpretation is often used in simulation of noise in real physical systems [16].

In Ref. [8] melting of an ultrathin lubricant film at friction between atomically flat mica surfaces has been represented as a result of action of spontaneously appearing shear stress leading to plastic flow. This is caused by heating of rubbing surfaces above critical value $T_{c 0}=1+g^{-1}$. The initial reason for self-organization process is the positive feedback of $T$ and $\sigma$ on $\varepsilon$ [see Eq. (3)]. This feedback is due to the temperature dependence of the shear viscosity leading to its divergence. On the other hand, the negative feedback of $\sigma$ and $\varepsilon$ on $T$ in Eq. (4) plays an important role since it ensures the system stability.

According to this approach, the lubricant represents a high-viscous liquid that can behave similar to the solid, i.e., has a high effective viscosity and still exhibits a yield stress $[3,12]$. The solid-like state of the lubricant corresponds to zero shear stress $(\sigma=0)$, because Eq. (2) falls out of consideration. Equation (3), which contains viscous stresses, reduces to the Debye law describing the relaxation of the shear strain during time $\tau_{\varepsilon}$. Finally, at $\lambda(t)=0$, heat conduction equation (4) takes the form of the simplest expression for temperature relaxation that does not contain the terms representing the dissipative heating and mechanocaloric effect of the viscous liquid.

Equation (3) describes the flow of lubricant with velocity $V=l \partial \varepsilon / \partial t$ due to the action of appearing viscous shear stress. Moreover, in accordance with Ref. [7], in the absence of shear deformations the temperature mean-square displacement is defined by equality $\left\langle u^{2}\right\rangle=T / G a$, where $a \sim 1 \mathrm{~nm}$ is the lattice constant or the intermolecular distance. The average shear displacement is found from the relationship $\left\langle u^{2}\right\rangle=\sigma^{2} a^{2} / G^{2}$. The total mean-square displacement represents the sum of these expressions provided that thermal fluctuations and stress are independent. The above implies that the transition of the lubricant from solid-like to fluid-like state is induced both by heating and under the effect of stress generated by solid surfaces in the course of friction. Such a view agrees with the examination of solid state instability within the framework of shear and dynamic disorder-driven melting representation in the absence of thermal fluctuations [7]. It is shown [19] that the plastic flow of a lubricant layer is realized when it contains elastic stresses. In this case, shear stresses decrease the shear modulus of the lubricant [20]. Consequently, 
the friction force decreases with increasing velocity at the contact $V=l \partial \varepsilon / \partial t$ because the latter leads to the growth of shear stress $\sigma$ according to the Maxwell stress - strain $\varepsilon$ relationship: $\partial \sigma / \partial t=-\sigma / \tau_{\sigma}+G \partial \varepsilon / \partial t$. It is assumed that the film becomes more liquid-like and the friction force decreases with the temperature growth due to decrease in activation energy barrier to molecular hops.

This work is devoted to study of the stochastic source $\lambda(t)$ effect on the evolution of stress $\sigma(t)$. In accordance with experimental data for organic lubricants $[3,7]$, the stress relaxation time under normal pressure is $\tau_{\sigma} \sim 10^{-10} \mathrm{~s}$, and it increases by several orders of magnitude under large pressures. Since the ultrathin lubricant film is less than four molecular layers thick, the temperature relaxes to $T_{e}$ during a time satisfying the inequality $\tau_{T} \ll \tau_{\sigma}$ [8]. Therefore, we will suppose that the adiabatic conditions are fulfilled

$$
\tau_{\sigma}, \tau_{\varepsilon} \gg \tau_{T},
$$

under which lubricant temperature $T$ follows the change of the shear components of stress $\sigma$ and strain $\varepsilon$. Then, one can separate out a small parameter and put $\tau_{T} \dot{T} \simeq 0$ in Eq. (4). As a result, we obtain an expression for the temperature:

$$
T=T_{e}-\sigma \varepsilon+\sigma^{2}+\lambda(t) .
$$

Let us simplify the system (2), (3), and (21) by reducing it for a single equation for shear stress $\sigma(t)$. To this end, $\varepsilon$ and $T$ should be expressed through $\sigma$. Differentiating an equation for strain $\varepsilon$ derived from (2) with respect of time, we get an equation for $\dot{\varepsilon}$. Substitution of these expressions for $\varepsilon$, $\dot{\varepsilon}$ and the equality (21) into (3) gives an evolutionary equation in the canonical form of equation for a nonlinear stochastic oscillator of the van der Pole generator type:

$$
m \ddot{\sigma}+\gamma(\sigma) \dot{\sigma}=f(\sigma)+\phi(\sigma) \lambda(t),
$$

where coefficient of friction $\gamma$, force $f$, amplitude of noise $\phi$, and parameter $m$ are defined by

$$
\begin{aligned}
& \gamma(\sigma) \equiv \frac{1}{g}\left[\tau_{\varepsilon}+\tau_{\sigma}\left(1+\sigma^{2}\right)\right], \quad f(\sigma) \equiv \sigma\left(T_{e}-1-g^{-1}\right)-\sigma^{3}\left(g^{-1}-1\right), \\
& \phi(\sigma) \equiv \sigma, \quad m \equiv \frac{\tau_{\sigma} \tau_{\varepsilon}}{g}
\end{aligned}
$$

\section{Effective Potential Method}

Let us find distribution of the system with time $t$ in the phase space that is parameterized by generalized coordinate $\sigma$ and momentum $p \equiv m \dot{\sigma}$. To this end, we will make use of the effective potential method that is described in this section on the basis of works [21,22]. Euler equation (22) is represented in the Hamiltonian form as follows:

$$
\dot{\sigma}=m^{-1} p, \quad \dot{p}=-m^{-1} \gamma(\sigma) p+f(\sigma)+\phi(\sigma) \lambda(t) .
$$

Statistical study is reduced to determination of function $\Pi(\sigma, p, t)$, which is the probability density of the presence of stress $\sigma$ and its derivative $\dot{\sigma}$ at given time 
instant $t$. It is averaged over the noise $\lambda$ distribution function $\rho(\sigma, p, t)$ of solutions to system (24):

$$
\Pi(\sigma, p, t)=\langle\rho(\sigma, p, t)\rangle .
$$

Let us assume that function $\rho=\rho(\sigma, p, t)$ satisfies the continuity equation

$$
\frac{\partial \rho}{\partial t}+\left[\frac{\partial}{\partial \sigma}(\dot{\sigma} \rho)+\frac{\partial}{\partial p}(\dot{p} \rho)\right]=0 .
$$

Plugging equations (24) into (26), we arrive at the Liouville equation

$$
\frac{\partial \rho}{\partial t}=(\widehat{L}+\widehat{N} \lambda) \rho
$$

with operators

$$
\widehat{L} \equiv-\frac{p}{m} \frac{\partial}{\partial \sigma}-\frac{\partial}{\partial p}\left(f-\frac{\gamma}{m} p\right), \quad \widehat{N} \equiv-\phi \frac{\partial}{\partial p} .
$$

Within the framework of the interaction representation a microscopic distribution function is defined by $\varpi=e^{-\widehat{L} t} \rho$, so that equation (27) assumes the form $\partial \varpi / \partial t=$ $\widehat{\mathcal{R}} \varpi$, where $\widehat{\mathcal{R}} \equiv \lambda\left(e^{-\widehat{L} t} \widehat{N} e^{\widehat{L} t}\right)$. The method of cumulant expansion [23] results in the linear differential kinetic equation $\partial\langle\varpi\rangle(t) / \partial t=\left[\int_{0}^{t}\left\langle\widehat{\mathcal{R}}(t) \widehat{\mathcal{R}}\left(t^{\prime}\right)\right\rangle \mathrm{d} t^{\prime}\right]\langle\varpi\rangle(t)$ with accuracy to the terms of order $O\left(\widehat{\mathcal{R}}^{2}\right)$. The transition from the interaction representation to the initial one gives for distribution function (25)

$$
\frac{\partial}{\partial t} \Pi(t)=\left\{\widehat{L}+\int_{0}^{t}\langle\lambda(\tau) \lambda(0)\rangle\left[\widehat{N}\left(e^{\widehat{L} \tau} \widehat{N} e^{-\widehat{L} \tau}\right)\right] \mathrm{d} \tau\right\} \Pi(t) .
$$

Since physical time $t$ is much longer that time of noise correlation $\tau_{\lambda}$, the upper limit of integration can be set equal to infinity. Then, expansion of exponents in (29) results in the expression

$$
\frac{\partial \Pi}{\partial t}=(\widehat{L}+\widehat{\Lambda}) \Pi
$$

where collision operator

$$
\widehat{\Lambda} \equiv \sum_{n=0}^{\infty} \widehat{\Lambda}^{(n)}, \quad \widehat{\Lambda}^{(n)} \equiv C^{(n)}\left(\widehat{N} \widehat{L}^{(n)}\right)
$$

is determined by commutators according to the recurrence formula

$$
\widehat{L}^{(n+1)}=\left[\widehat{L}, \widehat{L}^{(n)}\right], \quad \widehat{L}^{(0)} \equiv \widehat{N}
$$

$([\hat{A}, \hat{B}]=\hat{A} \hat{B}-\hat{B} \hat{A})$ and by the moments of correlation function (5)

$$
C^{(n)}=\frac{1}{n !} \int_{0}^{\infty} \tau^{n}\langle\lambda(\tau) \lambda(0)\rangle \mathrm{d} \tau
$$


The first moments are

$$
C^{(0)}=I, \quad C^{(1)}=I \tau_{\lambda} .
$$

In the general case, further consideration is impossible, therefore, we make the simplifying assumption, i.e., separate out small parameter $\epsilon \ll 1$, which coincides with the Kubo number [23]. Supposing $m=\epsilon^{2}$ in equation (22) and measuring the generalized momentum in units of $\epsilon$, we study the case of an overdamped oscillator in which the sliding friction force exceeds the other components by $\epsilon^{-1} \gg 1$ times. Then, equations (24) assume the form

$$
\frac{\partial \sigma}{\partial t}=\epsilon^{-1} p, \quad \frac{\partial p}{\partial t}=-\epsilon^{-2} \gamma(\sigma) p+\epsilon^{-1}[f(\sigma)+\phi(\sigma) \lambda(t)] .
$$

Accordingly, the Fokker-Plank equation (30) is written as

$$
\left(\frac{\partial}{\partial t}-\widehat{L}\right) \Pi=\epsilon^{-2} \widehat{\Lambda} \Pi
$$

where operator $\widehat{L} \equiv \epsilon^{-1} \widehat{L}_{1}+\epsilon^{-2} \widehat{L}_{2}$ has two components: $\widehat{L}_{1} \equiv-p(\partial / \partial \sigma)-f(\partial / \partial p)$ and $\widehat{L}_{2} \equiv \gamma(\partial / \partial p) p$. Operator of collision $\widehat{\Lambda}$ is defined by expressions (28), (31)(33) and with accuracy to terms of the second order of smallness the expansion (31) takes the form

$$
\begin{aligned}
\widehat{\Lambda} & =\left(C^{(0)}+\gamma C^{(1)}\right) \phi^{2} \frac{\partial^{2}}{\partial p^{2}} \\
& +\epsilon C^{(1)} \phi^{2}\left[-\frac{1}{\phi}\left(\frac{\partial \phi}{\partial \sigma}\right)\left(\frac{\partial}{\partial p}+p \frac{\partial^{2}}{\partial p^{2}}\right)+\frac{\partial^{2}}{\partial \sigma \partial p}\right]+O\left(\epsilon^{2}\right) .
\end{aligned}
$$

Since melting of the lubricant is characterized both by stress $\sigma$ and time $t$, we consider the projection of the distribution function on the half-space $(\sigma, t)$. To this end, let us pass to the Fokker-Plank equation relatively to function $P(\sigma, t)$, using the moments of the initial distribution $P_{n}(\sigma, t) \equiv \int p^{n} \Pi(\sigma, p, t) \mathrm{d} p$, the zeroth of which gives the desired result. Multiplying equation (36) by $p^{n}$ and integrating it over all momenta, we come to the recurrent relationship

$$
\begin{aligned}
\epsilon^{2} \frac{\partial P_{n}}{\partial t}-n \gamma P_{n} & +\epsilon\left(\frac{\partial P_{n+1}}{\partial \sigma}+n f P_{n-1}\right)=n(n-1)\left(C^{(0)}+\gamma C^{(1)}\right) \phi^{2} P_{n-2} \\
& +\epsilon n C^{(1)}\left[\phi^{2} \frac{\partial P_{n-1}}{\partial \sigma}-n \phi\left(\frac{\partial \phi}{\partial \sigma}\right) P_{n-1}\right]+O\left(\epsilon^{2}\right) .
\end{aligned}
$$

Further, the Fokker-Plank equation is derived on the basis of the hierarchical approach to (38). At $n=0$, we obtain an equation for desired function $P=P_{0}(\sigma, t)$ :

$$
\frac{\partial P}{\partial t}=-\epsilon^{-1} \frac{\partial P_{1}}{\partial \sigma} .
$$

First-order moment $P_{1}$ is fixed by the equation

$$
P_{1}=\frac{\epsilon}{\gamma}\left\{f P+\frac{\partial P_{2}}{\partial \sigma}-C^{(1)}\left[\phi^{2} \frac{\partial P}{\partial \sigma}-\phi\left(\frac{\partial \phi}{\partial \sigma}\right) P\right]\right\}
$$


following from (38) where $n=1$ and terms of the first order of smallness in $\epsilon \ll 1$ are taken into account. Consideration of terms of zeroth order in $\epsilon$ at $n=2$ allows us to write an expression for the second-order moment $P_{2}$ :

$$
P_{2}=-\left(\frac{C^{(0)}}{\gamma}+C^{(1)}\right) \phi^{2} P .
$$

As a result, the Fokker-Planck equation

$$
\frac{\partial P}{\partial t}=-\frac{\partial}{\partial \sigma}\left(D^{(1)} P\right)+\frac{\partial}{\partial \sigma}\left(D^{(2)} \frac{\partial P}{\partial \sigma}\right)
$$

is obtained. It is expressed in terms of coefficients

$$
D^{(1)}=\frac{1}{\gamma}\left\{f-I \phi^{2} \frac{\partial \gamma^{-1}}{\partial \sigma}-\phi \frac{\partial \phi}{\partial \sigma}\left(\frac{2 I}{\gamma}+I \tau_{\lambda}\right)\right\}, \quad D^{(2)}=\frac{\phi^{2}}{\gamma}\left(\frac{I}{\gamma}+2 I \tau_{\lambda}\right) .
$$

In the stationary case, the solution of Eq. (42) leads to the distribution

$$
P(\sigma)=\mathcal{Z}^{-1} \exp \{-E(\sigma)\}
$$

which is fixed by an effective potential

$$
E(\sigma)=-\int_{0}^{\sigma} \frac{D^{(1)}(x)}{D^{(2)}(x)} \mathrm{d} x
$$

and a normalization constant $\mathcal{Z}=\int_{0}^{\infty} \mathrm{d} \sigma \exp \{-E(\sigma)\}$.

\section{Continuous Transition}

The stationary shear stress is found from an extremum condition of distribution (44)

$$
\frac{D^{(1)}(\sigma)}{D^{(2)}(\sigma)}=0
$$

Substituting expressions (43), (23) into (46), we arrive at the equation

$$
\left\{T_{e}-\frac{1+g}{g}-\sigma^{2}\left(\frac{1-g}{g}\right)-I \tau_{\lambda}\right\}\left[\tau_{\varepsilon}+\tau_{\sigma}\left(1+\sigma^{2}\right)\right]^{2}-2 I g\left(\tau_{\varepsilon}+\tau_{\sigma}\right)=0 .
$$

According to (47) and Fig. 3, distribution (44) has pronounced maxima whose positions are determined by the set of parameters $\tau_{\sigma}, \tau_{\varepsilon}, \tau_{\lambda}, g, I$, and $T_{e}$.

At small values of the friction surfaces temperature $T_{e}$ a single maximum is realized at point $\sigma=0$ corresponding to the dry friction mode (curve 1). With $T_{e}$ growth two maxima appear at points $\sigma=0$ and $\sigma \neq 0$ (curve 2), the first peak corresponds to the dry friction, the second one to the sliding. Here the stick-slip friction mode, which is characterized by transitions between the indicated stationary regimes, is realized. With the further growth of $T_{e}$ the zeroth maximum of $P(\sigma)$ 


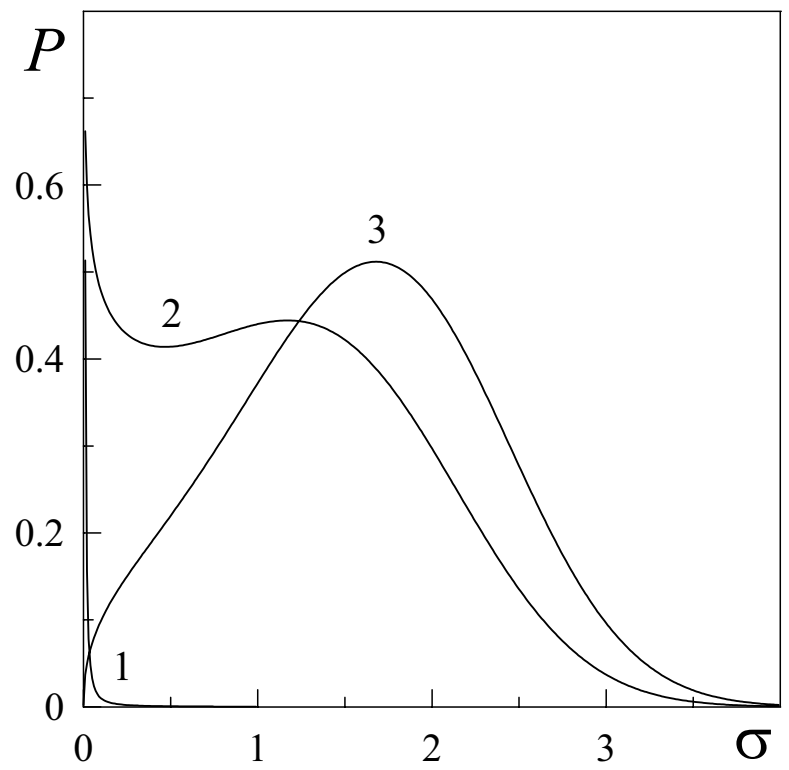

Fig. 3. Distribution function of shear stress (44) for the second-order transition at $g=0.2$, $\tau_{\sigma}=\tau_{\varepsilon}=0.1, \tau_{\lambda}=0.2$, and $I=5$. Curves $1,2,3$ correspond to the temperatures $T_{e}=5,16,20$, respectively.

disappears and the maximum at $\sigma \neq 0$ remains only (curve 3), i.e., the lubricant becomes liquid-like.

Supposing in (47) $\sigma=0$, we find a critical value of the friction surfaces temperature

$$
T_{e 0}=\frac{1+g}{g}+\left(\tau_{\lambda}+\frac{2 g}{\tau_{\varepsilon}+\tau_{\sigma}}\right) I
$$

providing the transition to sliding mode. It is seen that $T_{e 0}$ grows with noise intensity $I$ and correlation time $\tau_{\lambda}$. The relaxation times of shear stress and strain influence $T_{e 0}$ inversely. The domains of the dry (DF), sliding (SF), and stick-slip (SS) modes of friction are realized in the phase diagram presented in Fig. 4. The growth of correlation time $\tau_{\lambda}$ results in increasing in the value $T_{e}$ corresponding to tricritical point $T$ at given intensity $I$. Thus, the region of the dry friction expands, while the probability of the sliding and stick-slip friction decreases.

Apparently, increase in the sheared surfaces temperature $T_{e}$ transforms the lubricant to the liquid-like state. It can be understood from Eq. (22) that describes damped oscillations. Here the surfaces temperature is included only in expression for the driving force $f$, which increases with growth of $T_{e}$. As it is seen from the phase portraits in Fig. 5 the liquid state corresponds to an oscillation mode with large amplitude. With increasing in the effective force in (22) the amplitude of oscillations increases, and the longer oscillation process is realized till setting of a certain friction mode in the system.

In studying a dynamics of change of friction modes we represent the distribution $P(\sigma)$ by position of its maximum $\tilde{\sigma}$. This is achieved by using the path integrals 


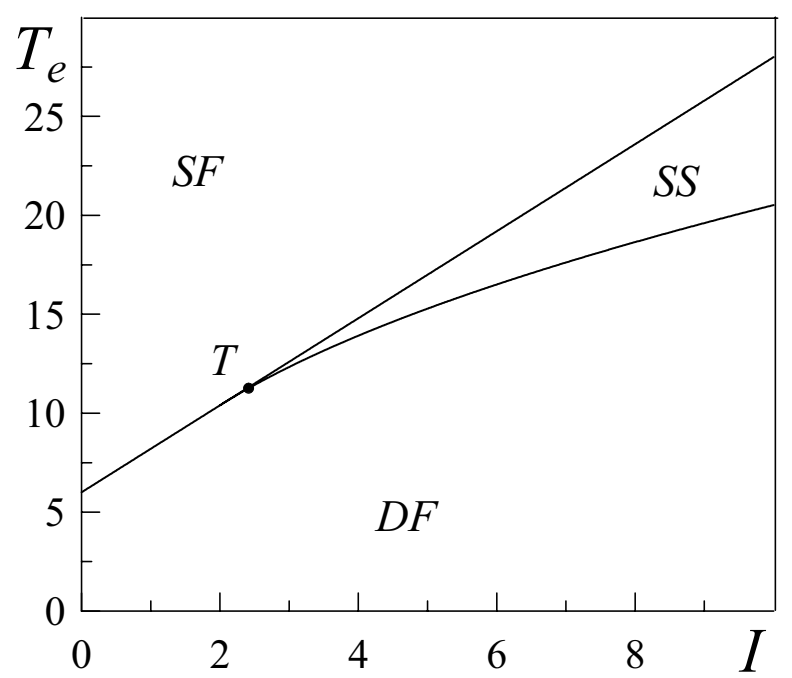

Fig. 4. Phase diagram corresponding to the parameters of Fig. 3 with the domains of the dry (DF), sliding (SF), and stick-slip (SS) friction ( $T$ is the tricritical point).

formalism [24]. Within the framework of such approach the extreme values $\tilde{\sigma}=$ $\tilde{\sigma}(t)$ of the initial distribution function (44) evolve in accordance with an effective distribution

$$
\Pi\{\dot{\tilde{\sigma}}, \tilde{\sigma}\} \propto \exp \left(-\int \Lambda(\dot{\tilde{\sigma}}, \tilde{\sigma}, t) \mathrm{d} t\right) .
$$

Here the Onsager-Machlup function $\Lambda$, acting as the Lagrangian of the Euclidean field theory, is the subject for determination.

Equation (42) can be transformed to the Ito form:

$$
\frac{\partial P}{\partial t}=-\frac{\partial}{\partial \sigma}\left[\left(D^{(1)}+\frac{\mathrm{d} D^{(2)}}{\mathrm{d} \sigma}\right) P\right]+\frac{\partial^{2}}{\partial \sigma^{2}}\left(D^{(2)} P\right) .
$$

In order to find the $\Lambda(\dot{\tilde{\sigma}}, \tilde{\sigma}, t)$ dependence, we write the differential Langevin equation

$$
\mathrm{d} \tilde{\sigma}=\left(D^{(1)}+\frac{\mathrm{d} D^{(2)}}{\mathrm{d} \sigma}\right) \mathrm{d} t+\sqrt{2 D^{(2)}} \mathrm{d} W(t)
$$

corresponding to the Fokker-Planck equation (50) [24]. Here a stochastic differential $\mathrm{d} W(t)$ represents the Winner process with the properties of the white noise:

$$
\langle\mathrm{d} W(t)\rangle=0, \quad\left\langle(\mathrm{~d} W(t))^{2}\right\rangle=\mathrm{d} t .
$$

The feature of stochastic equations is that the differential $\mathrm{d} W(t)$ can not be obtained by a simple division of Eq. (51) by $\sqrt{2 D^{(2)}}$. To this end, one should pass from the random process $\tilde{\sigma}(t)$ to the white noise $x(t)$ related with the initial Jacobian $\mathrm{d} x / \mathrm{d} \tilde{\sigma}=\left(2 D^{(2)}\right)^{-1 / 2}$. Then, substitution of Eq. (51) into the Ito stochastic differential

$$
\mathrm{d} x=\frac{\mathrm{d} x}{\mathrm{~d} \tilde{\sigma}} \mathrm{d} \tilde{\sigma}+\frac{1}{2} \frac{\mathrm{d}^{2} x}{\mathrm{~d} \tilde{\sigma}^{2}}(\mathrm{~d} \tilde{\sigma})^{2},
$$


taking into account (52), leads to the expression

$$
\mathrm{d} x=\left(\frac{\mathrm{d} x}{\mathrm{~d} \tilde{\sigma}}\left(D^{(1)}+\frac{\mathrm{d} D^{(2)}}{\mathrm{d} \tilde{\sigma}}\right)+\frac{\mathrm{d}^{2} x}{\mathrm{~d} \tilde{\sigma}^{2}} D^{(2)}\right) \mathrm{d} t+\frac{\mathrm{d} x}{\mathrm{~d} \tilde{\sigma}} \sqrt{2 D^{(2)}} \mathrm{d} W(t) .
$$

Here the terms, whose order exceeds $(\mathrm{d} W(t))^{2}$, are neglected. After reverse transition from the white noise $x(t)$ to the initial process $\tilde{\sigma}(t)$ the equality

$$
\frac{\mathrm{d} W(t)}{\mathrm{d} t}=\frac{\dot{\tilde{\sigma}}}{\sqrt{2 D^{(2)}}}-\frac{2 D^{(1)}+\left(D^{(2)}\right)^{\prime}}{2 \sqrt{2 D^{(2)}}}
$$

is obtained, where the stroke stands for differentiation with respect to $\tilde{\sigma}$. Plugging this expression into the Gaussian

$$
\Pi \propto \exp \left\{-\frac{1}{2} \int\left(\frac{\mathrm{d} W(t)}{\mathrm{d} t}\right)^{2} \mathrm{~d} t\right\}
$$

and comparing with (49), we arrive at the Lagrangian

$$
\Lambda=\frac{1}{4} \frac{\dot{\tilde{\sigma}}^{2}}{D^{(2)}}-U
$$

with a potential energy

$$
U=-\frac{\left(2 D^{(1)}+\left(D^{(2)}\right)^{\prime}\right)^{2}}{16 D^{(2)}} .
$$

It is substantial that such form of the potential energy $U$ does not coincide with the effective potential (45). Consequently, for further consideration one should replace the expression $U(57)$ by $E(\sigma)$ (45). In this case, Eq. (56) describes the system behaviour in accordance with the distribution (44) that is obtained above.

The system kinetics is defined by the Euler-Lagrange equation

$$
\frac{\partial \Lambda}{\partial \tilde{\sigma}}-\frac{\partial}{\partial t} \frac{\partial \Lambda}{\partial \dot{\tilde{\sigma}}}=\frac{\partial R}{\partial \dot{\tilde{\sigma}}}
$$

Within the white noise representation a dissipative function has the simplest form $R=\dot{x}^{2} / 2$. It is transformed to

$$
R=\frac{\dot{\tilde{\sigma}}^{2}}{4 D^{(2)}}
$$

at transition to a variable $\dot{\tilde{\sigma}}=\left(2 D^{(2)}\right)^{1 / 2} \dot{x}$. Substituting the equalities (56), (45), and (59) into (58), we come to the differential equation

$$
\ddot{\tilde{\sigma}}+\dot{\tilde{\sigma}}^{2} \frac{\left(D^{(2)}\right)^{\prime}}{2 D^{(2)}}+\dot{\tilde{\sigma}}-2 \frac{D^{(1)}}{D^{(2)}} D^{(2)}=0 .
$$

Supposing in (60) $\dot{\tilde{\sigma}}=0$ at the steady state the extremum condition (46) of distribution (44) is obtained. Note that the distribution maximum corresponds to the minimum of effective potential, and the distribution minimum to the potential maximum. 


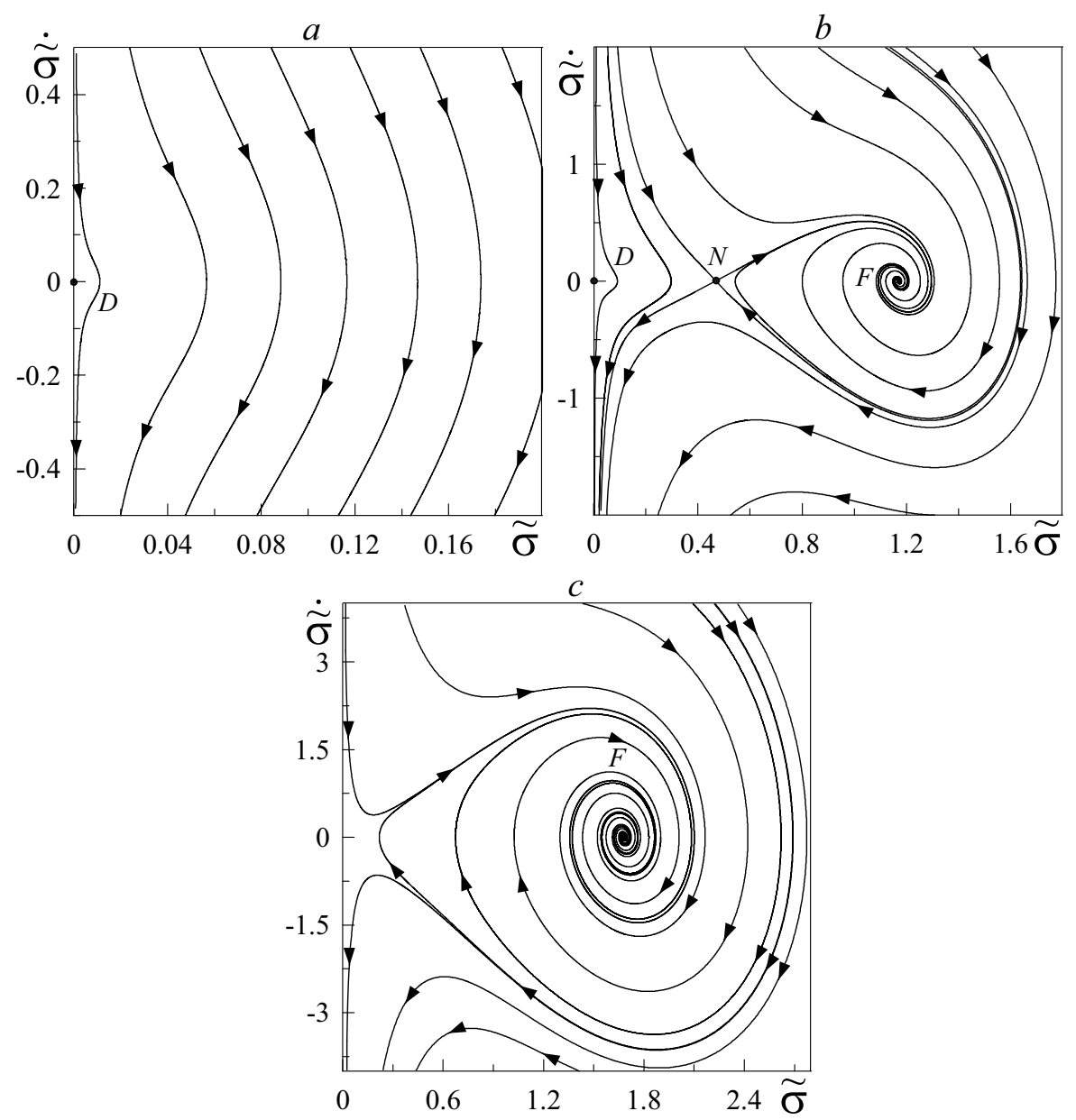

Fig. 5. Phase portraits corresponding to the parameters of Fig. 3. a: DF mode corresponds to the curve 1 in Fig. 3 . b: SS mode to the curve 2 in Fig. 3 . c: SF mode to the curve 3 in Fig. 3.

Consider kinetics of the system using the phase plane method for analysis of Eq. (60). The phase portraits, corresponding to the curves of Fig. 3, are presented in Fig. 5.

The region of the dry friction (DF) (Fig. 5a) is characterized by the presence of one singular point $D$ that corresponds to the maximum of probability $P(\sigma)$ at $\sigma=0$. This point is non-standard and it requires interpretations. It is located at the origin of coordinates and phase trajectories are curved around it, so that the system never comes to the value $\sigma=0$, i.e., this point is unstable stationary. Consider the system behaviour at the arbitrary initial condition. According to the phase trajectories, the system evolves to the zero value of stress. Thus, if at initial conditions the growth rate of stress is positive it decreases to the zero at first (during this time the stress increases). Then, the stress decreases asymptotically to the zero with increase in its decreasing rate. Presumably, such situation corresponds to the 
mode of explosive amorphization, in which the system very rapidly transforms in an amorphous solid-like state. The zero stress is never achieved due to the divergence of the probability $P(\sigma)$ at point $\sigma=0$. This is related with the infinite growth of decrease rate of stress variation at motion of the system to $\sigma=0$. Let us assume that the system has reached the point, at which decreasing rate takes a critical value. In such case, the increase in stress value is expected, which is accompanied with the change of rate sign and transition of the system in the positive phase plane domain. Further, the decreasing in stress occurs again, and the described situation repeats itself. Alternatively, the sign of stress variation rate does not change, and only its decreasing takes place. This moves the system on the neighboring phase trajectory, along which it approaches to the zero stress more rapidly. As a result, in the course of time the oscillation mode of dry friction sets in nearby the point $\sigma=0$ at the arbitrary initial conditions. Thus, in this case, the oscillations amplitude is small and the lubricant has the solid-like structure.

The phase portrait of the system, characterizing the region of the stick-slip friction (SS), is shown in Fig. 5b. Three singular points appear here: $D$, a saddle $N$, and a stable focus $F$. As well as above, the point $D$ is realized at the origin of coordinates and it corresponds to the dry friction mode. The saddle $N$ characterizes the minimum of $P(\sigma)$ and it is the unstable stationary point. It is worth noting that at the initial value of shear stress on the right-hand side from the point $N$ and $\dot{\sigma}=0$, the sliding mode of friction sets in as evolution proceeds. If the initial value of stress appears on the left-hand side from saddle $N$, in the similar case, the dry friction sets in. Thus, the point $N$ separates two maxima of the distribution function $P(\sigma)$. The focus $F$ corresponds to the non-zero maximum of the stress distribution function, i.e., it describes the liquid-like state of the lubricant. The damped oscillations, corresponding to this point, mean that lubricant becomes more liquid-like and more solid-like periodically. However, the stable sliding friction always sets in. Presumably, these oscillations are conditioned by the presence of correlated noise.

The phase portrait, describing the sliding friction $(\mathrm{SF})$, which is characterized by single non-zero maximum of the distribution function $P(\sigma)$, is shown in Fig. 5c. It has one singular point (the stable focus $F$ ). Stability of sliding friction is also confirmed by large expansion of the phase trajectories near $F$ along the axes of coordinates. However, it is apparent that at the initial large value of $\sigma$ the system does not reach the point $F$, and it approaches asymptotically to the zero stress. This circumstance implies that conditions, under which the system will be near the regime of dry friction, can be realized. As described above, after reaching the critical variation rate of stress its sign changes and becomes positive. It is seen from the phase portrait that, in such case, the system also passes to the mode of stable sliding friction.

\section{Effect of Deformation Defect of Modulus}

Actually, the shear modulus of the lubricant, introduced (in terms of the relaxation time $\tau_{\sigma}$ ) in Eq. (2), depends on the stress value $[19,20]$. This leads to the transition

of elastic deformation mode to plastic one. It takes place at characteristic value of the stress $\sigma_{p}$, which does not exceed $\sigma_{s}$ (in other case, plastic flow is not manifested). 
For consideration of deformation defect of the modulus we will make use of the dependence $\tau_{\sigma}(\sigma)$ proposed in [8], instead of using stress-independent $\tau_{\sigma}$. As a result, Eq. (2) takes the form:

$$
\tau_{p} \dot{\sigma}=-\sigma\left(1+\frac{\theta^{-1}-1}{1+\sigma / \alpha}\right)+g_{\Theta} \varepsilon
$$

where the relaxation time for the plastic mode $\tau_{p}=\eta_{\sigma} / \Theta$ is introduced $\left(\eta_{\sigma} \equiv \tau_{\sigma} G\right.$ is the effective viscosity, $\Theta$ is the hardening factor), $\theta=\Theta / G<1$ is the parameter describing the ratio of tilts of plastic and Hookean sections of the deformation curve, $g_{\Theta}=G^{2} / \Theta G_{0}$ and $\alpha=\sigma_{p} / \sigma_{s}$ are constants. Then, within the framework of approximation (20), the system (61), (3), and (4), as well as above, is reduced to Eq. (22), where coefficient of friction $\gamma$, force $f$, amplitude of noise $\phi$, and parameter $m$ are defined by

$$
\begin{aligned}
\gamma(\sigma) & \equiv \frac{1}{g}\left[\tau_{\varepsilon}\left(1+\frac{\theta^{-1}-1}{(1+\sigma / \alpha)^{2}}\right)+\tau_{p}\left(1+\sigma^{2}\right)\right], \\
f(\sigma) & \equiv \sigma\left[T_{e}-1-\frac{1}{g}\left(\frac{\theta^{-1}+\sigma / \alpha}{1+\sigma / \alpha}\right)\right]-\sigma^{3}\left[\frac{1}{g}\left(\frac{\theta^{-1}+\sigma / \alpha}{1+\sigma / \alpha}\right)-1\right], \\
\phi(\sigma) & \equiv \sigma, \quad m \equiv \frac{\tau_{p} \tau_{\varepsilon}}{g_{\Theta}}
\end{aligned}
$$

According to the effective potential method, described in Section 3, we obtain the Fokker-Planck equation (42) with corresponding coefficients $D^{(1)}$ and $D^{(2)}$. (47):

Using Eqs. (46), (43), and (62) the equation is found with the same meaning as

$$
\begin{aligned}
& \left\{T_{e}-1-\frac{1}{g_{\Theta}}\left(\frac{\theta^{-1}+\sigma / \alpha}{1+\sigma / \alpha}\right)-\sigma^{2}\left[\frac{1}{g_{\Theta}}\left(\frac{\theta^{-1}+\sigma / \alpha}{1+\sigma / \alpha}\right)-1\right]-I \tau_{\lambda}\right\} \gamma^{2} \\
& -2 I \gamma+\frac{2 I \sigma}{g_{\Theta}}\left\{\tau_{p} \sigma-\tau_{\varepsilon} \frac{\theta^{-1}-1}{\alpha(1+\sigma / \alpha)^{3}}\right\}=0 .
\end{aligned}
$$

It gives the boundary of existence of distribution (44) maximum at zero value of stress corresponding to the solid-like state of the lubricant

$$
T_{e 0}=\frac{\theta^{-1}+g_{\Theta}}{g_{\Theta}}+\left(\tau_{\lambda}+\frac{2 g_{\Theta}}{\theta^{-1} \tau_{\varepsilon}+\tau_{p}}\right) I .
$$

The dependencies $P(\sigma)$ for different modes of friction are shown in Fig. 6 .

Curves $1-5$ correspond to the domains of the dry (DF), stick-slip (SS), stick-slip and sliding $(\mathrm{SS}+\mathrm{SF})$, metastable and stable sliding $(\mathrm{MSF}+\mathrm{SF})$, and sliding friction $(\mathrm{SF})$, respectively. The phase diagram and portraits are presented in Figs. 7 and 8 corresponding to the curves of Fig. 6.

The most complex form of the function $P(\sigma)$ is inherent in the SS+SF region (curve 3 in Fig. 6). Here the solid-like, metastable and stable liquid-like lubricant states coexist, which corresponds to the maxima of $P(\sigma)$. It means the realization possibility of intermittent (stick-slip) friction, in which the periodic transitions occur between the dynamic modes corresponding to these states. In the MSF+SF domain 


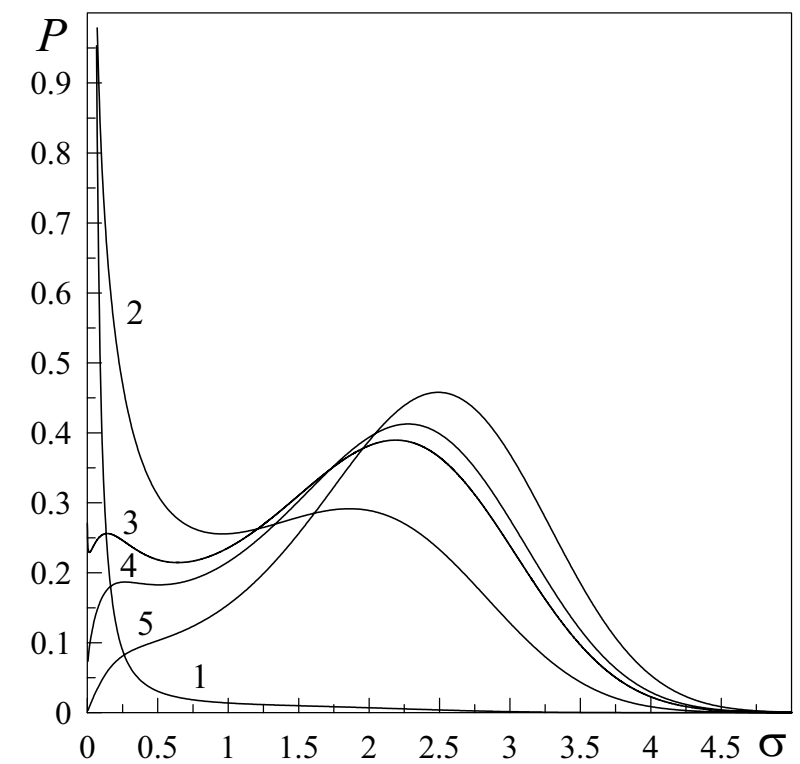

Fig. 6. Distribution function of shear stress (44) for the first-order transition at $\tau_{p}=\tau_{\varepsilon}=\tau_{\lambda}=$ $0.1, \theta^{-1}=7, \alpha=0.3, g_{\Theta}=0.4, I=4.5$. Curves $1-5$ correspond to the temperatures $T_{e}=16,21,23.25,24,26$, respectively.

of stick-slip motion the metastable and stable sliding can periodically pass into each other (curve 4 in Fig. 6). Remarkably, that transition from the SS+SF to MSF+SF region is accompanied with disappearance of dry friction in the system. The domain of the dry friction (DF) expands, and the region of the sliding friction (SF) shrinks with growth of the correlation time $\tau_{\lambda}$ of noise.

The phase portrait of the dry friction region (DF) is similar to that is inherent in continuous transformation (Fig. 5a). It implies that the DF domain is equivalent taking into account the modulus defect and without it.

The phase portrait, describing the region of the stick-slip friction (SS), is similar to that shown in Fig. 5b for continuous transformation. Their basic difference is that, in this case, the trajectories around the focus are considerably more elongated along both coordinates axes. It means the greater stability of sliding friction.

The most complex region ( $\mathrm{SS}+\mathrm{SF}$ ) is represented in the phase portrait (Fig. 8a). Five singular points are realized here: $D$, the saddles $N, N^{\prime}$, the stable focuses $F$, $F^{\prime}$. As well as above, the saddles correspond to the minima of dependence $P(\sigma)$. Point $D$ describes the solid-like state of the lubricant. Stable focus $F$ determines the first non-zero maximum of probability. It is apparent that the oscillations are weakly pronounced around this point. In this mode the lubricant represents a highviscous liquid, and in such type of fluid the oscillations are strongly damped at the presence of correlated noise. Actually, the point $F$ corresponds to the small values of stress. With decrease in $\sigma$ the lubricant becomes more viscous, and it is transformed into the solid-like state at $\sigma=0$. Focus $F^{\prime}$ describes the second non-zero maximum of the function $P(\sigma)$, and there are oscillations with large amplitude around it. 


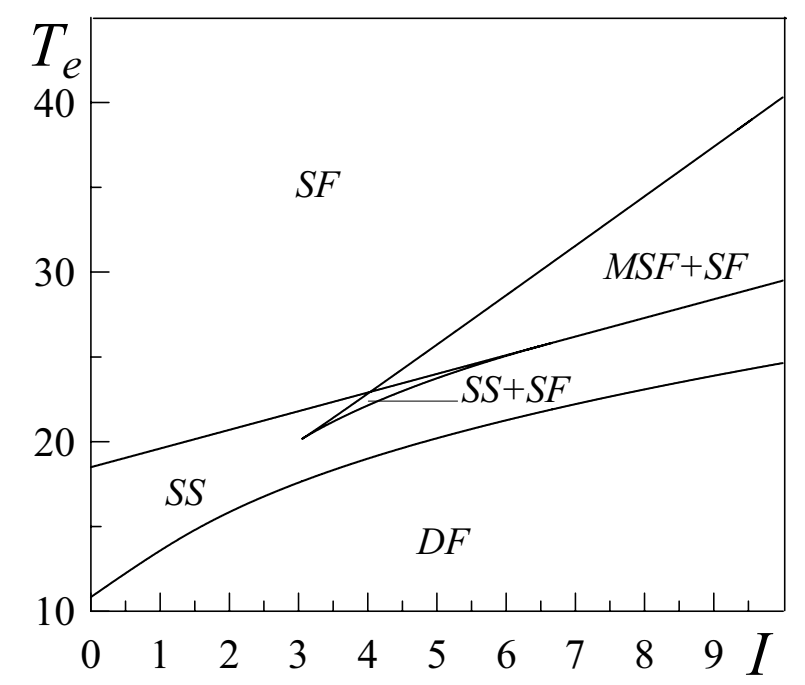

Fig. 7. Phase diagram corresponding to the parameters of Fig. 6 with the domains of the dry $(\mathrm{DF})$, sliding $(\mathrm{SF})$, and stick-slip (SS, MSF+SF, SS+SF) friction modes.

Such behaviour corresponds to the fluid-like state of the lubricant and, accordingly, sliding. The point $F^{\prime}$ is located on large distance along abscissa axis from all other ones. This mode of friction is only the most probable, since points $D$ and $F$, corresponding to the dry and metastable sliding friction, also have large stability and probability of realization. So we can draw the conclusion that the lubricant can experience periodic transitions (stick-slip) between the friction modes corresponding to the points $D, F$, and $F^{\prime}$. Since these modes are stable and separated by the pronounced minima of the distribution function $P(\sigma)$ (by the saddles), one should expect transitions between them on the expiry of long intervals of time.

The phase portrait of the MSF+SF domain is represented in Fig. 8b. There are three singular points: the stable focuses $F, F^{\prime}$, and the saddle $N$. The latter is similar to the saddles described above and it characterizes the minimum of probability dependence on the stress. Point $F$ corresponds to the first maximum of the distribution, which describes the metastable sliding mode (MSF), and $F^{\prime}$ meets the second maximum, which defines the stable sliding (SF). There are only insignificant oscillations around the focus $F$ however, the lubricant in this mode is less viscous liquid than in the vicinity of the point $F$ in Fig. 8a. At the origin of coordinates the singular point is absent and the dry friction is not realized. Focus $F^{\prime}$ is similar to that described in Fig. 8a however, its "attraction" domain is more stretched along the both coordinates axes that means the larger stability of this mode. Therefore, in comparison with the previous case, arising of the sliding friction is more probable here.

The SF region is represented by the phase portrait, which is similar to that described for the continuous transformation (Fig. 5c). Here the one stable focus $F$ is realized and the oscillations represent the stable sliding friction. The basic difference, in this case, is that the oscillations take place with larger amplitude. 


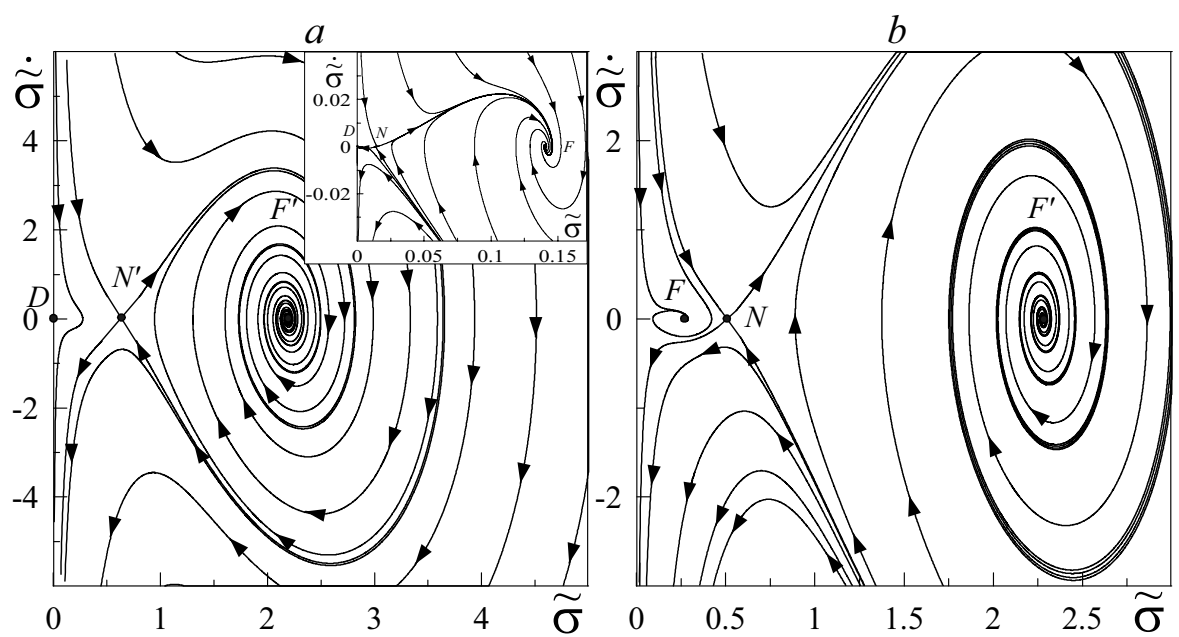

Fig. 8. Phase portraits corresponding to the parameters of Fig. 6. a: SS+SF mode corresponds to the curve 3 in Fig. 6 . b: MSF+SF mode to the curve 4 in Fig. 6.

This implies stability of such mode. However, as well as in all above considered situations, the stationary shear stress, corresponding to the maximum of the initial distribution $P(\sigma)$, sets in as evolution proceeds.

In the basic equations (2) - (4) the shear stress $\sigma$ has the first power. However, in general case, its exponent $a$ may be fractional $(0<a<1)$, but not integer:

$$
\begin{aligned}
\tau_{\sigma} \dot{\sigma} & =-\sigma^{a}+g \varepsilon, \\
\tau_{\varepsilon} \dot{\varepsilon} & =-\varepsilon+(T-1) \sigma^{a}, \\
\tau_{T} \dot{T} & =\left(T_{e}-T\right)-\sigma^{a} \varepsilon+\sigma^{2 a}+\lambda(t) .
\end{aligned}
$$

Taking into account the additive noises of the shear stress and strain, and the temperature of the lubricant film it is shown [10] that such system describes the self-similar mode for which the characteristic scale of shear stress is absent [25]. This regime is determined by the homogeneous distribution function

$$
P(y)=y^{-2 a} \mathcal{P}(\sigma), \quad y=\sigma \sigma_{s} .
$$

In particular, the value $2 a=1.5$ corresponds to a self-organized criticality mode, at which the process of self-organization (lubricant melting) does not require the external influence $\left(T_{e}=0\right)$ and it occurs spontaneously $[10,26]$, unlike the phase transition.

The self-similar behaviour of the lubricant film is studied taking into account fluctuations of its temperature defined by the Ornstein-Uhlenbeck process. It is shown that the fluctuations of the lubricant temperature result in disappearance of the sliding friction region at the presence of the dry and stick-slip friction domains in both cases of second- and first-order transitions. In the latter case, the stick-slip motion arises, that is characterized by three stationary values of shear stresses, at which the dry, metastable and stable sliding friction are realized. The increase in 
the correlation time of the lubricant temperature fluctuations leads to increasing in the rubbing-surface temperature needed for realization of stick-slip friction.

The study of Eqs. (65) - (67) shows that the phase portraits for the similar domains reproduce above considered qualitatively. However, there are substantial differences. The fractional Lorentz system at $a \neq 1$ and $I \neq 0$ results in the presence of the singular point $D$, which corresponds to the solid-like state of the lubricant, in the phase portraits. Besides, variation of $a$ leads to a complication of the dependence $P(\sigma)$, and, as a result, to a more complex form of phase portraits. Within the determined friction mode at decreasing in $a$ increase in the abscissas of the stable focuses is observed. Consequently, the weakening of fractional feedbacks in a Lorentz-type models results in increasing in the lubricant fluidity and the reduction of friction. However, in the systems described by fractional exponent $a$ the dry friction is always realized. Thus, it is impossible to assert that such systems are more preferable to friction decrease than the linear systems.

\section{Conclusion}

The above consideration shows that the increase in the temperature of rubbing surfaces $T_{e}$, at the presence of correlated noise of the lubricant temperature, can be accompanied with self-organization of deformation and thermal fields leading to the mode of sliding friction. Indeed, the correlation degree of the lubricant temperature change plays a substantial role. If the correlation time $\tau_{\lambda}$ increases at a fixed intensity $I$ of temperature fluctuations, the friction surfaces temperature must grow for the transition from the dry to sliding friction mode. In the case of continuous transformation at small intensity $I$, this transition takes place without forming the region of intermittent friction, i.e., proceeds as the second-order transition (melting of the amorphous lubricant). In the reverse case of large $I$, the first-order transition (melting of the crystalline lubricant) is realized.

At setting of the sliding friction mode in the system the damped oscillations arise and the shear stress relaxes to the stationary value that is fixed by the probability distribution $P(\sigma)$. The amplitude of these oscillations increases with growth of the stationary values of shear stress corresponding to the liquid-like structure of the lubricant. The solid-like state of the lubricant is described by the singular point $D$ at the origin of coordinates that has complex character of stability. It is characterized by the divergence of probability $P(\sigma)$. The oscillations near this point are possible.

For description of the first-order transition the shear modulus defect is taken into account. It is shown that a change in the temperature fluctuation intensity $I$ and/or in the rubbing-surface temperature $T_{e}$ may transform the system from the dry friction mode to the sliding one. The latter arises at two values of the shear stress. Accordingly, three singular points, which define the stationary values of stress, appear in the phase portraits: the non-standard point $D$ at zero stress and two stable focuses at non-zero stresses. The intermittent (stick-slip) mode of friction can be realized as a result of transitions between the solid-like, metastable and stable liquid-like lubricant states, which are described by the zero and non-zero singular points.

Taking into consideration the nonlinear relaxation of the shear stress and fractional feedbacks in the Lorentz system it is shown that the region of the sliding 
friction is realized in the phase diagram only in the absence of temperature fluctuations. In this case, the singular point $D$, that corresponds to the solid-like state of the lubricant and the dry friction, is always present in phase portraits. Besides, the change of fractional exponent can make the phase diagram more complicated due to increasing in the number of friction domains in accordance with experimental data $[3]$.

\section{Acknowledgements}

We thank Dr. O. V. Boyko and A. S. Kornyushchenko for attentive reading and correction of the manuscript. We express our gratitude to the organizers and participants of Working conference-seminar of Institute for Condensed Matter Physics of National Academy Sciences of Ukraine (2-3 of June, 2005, Lviv) within the framework of the fifth Ukrainian competition of young scientists in the field of statistical physics and theory of condensed medium for discussing and supporting the work. The work was partly supported by a grant of the cabinet of Ukraine.

\section{References}

[1] B. N. J. Persson, Sliding Friction. Physical Principles and Applications, SpringerVerlag, Berlin (1998).

[2] F. P. Bowden, D. Tabor, The Friction and Lubrication of Solids, Clarendon Press, Oxford (1985).

[3] H. Yoshizawa, Y.-L. Chen, J. Israelachvili, Fundamental Mechanisms of Interfacial Friction. 1. Relation between Adhesion and Friction, J. Phys. Chem. 97 (1993) 41284140; H. Yoshizawa, J. Israelachvili, Fundamental Mechanisms of Interfacial Friction. 2. Stick-Slip Friction of Spherical and Chain Molecules, J. Phys. Chem. 97 (1993) 11300-11313;

[4] O. M. Braun, A. G. Naumovets, Nanotribology: Microscopic mechanisms of friction, Surface Science Reports 60 (2006) 79-158.

[5] J. Krim, D. H. Solina, R. Chiarello, Nanotribology of a Kr monolayer: A quartz-crystal microbalance study of atomic-scale friction, Phys. Rev. Lett. 66 (1991) 181-184.

[6] J. M. Carlson, A. A. Batista, Constitutive relation for the friction between lubricated surfaces, Phys. Rev. E 53 (1996) 4153-4165.

[7] I. S. Aranson, L. S. Tsimring, V. M. Vinokur, Stick-slip friction and nucleation dynamics of ultrathin liquid films, Phys. Rev. B 65 (2002) 125402-7.

[8] A. V. Khomenko, O. V. Yushchenko, Solid-liquid transition of ultrathin lubricant film, Phys. Rev. E 68 (2003) 036110-6.

[9] A. V. Khomenko, Noise influence on solid-liquid transition of ultrathin lubricant film, Phys. Lett. A 329 (2004) 140-147.

[10] A. V. Khomenko, I. A. Lyashenko, Stochastic theory of ultrathin lubricant film melting in the stick-slip regime, Technical Physics 50 (2005) 1408-1416; A. I. Olemskoi, A. V. Khomenko, D. O. Kharchenko, Self-organized Criticality within Fractional Lorenz Scheme, Physica A 323 (2003) 263-293.

[11] A.V. Khomenko, I.A. Lyashenko, Temperature dependence effect of viscosity on ultrathin lubricant film melting, Condensed Matter Physics 9, No. 4(48) (2006) 695-702.

[12] Rheology, eds. F. R. Eirich, Academic Press, New York (1960).

[13] J. Jäckle, Models of the glass transition, Rep. Prog. Phys. 49 (1986) 171-231; A. Havranek, M. Marvan, Glass transition in inorganic glasses, polymers, and crystals, Ferroelectrics 176 (1996) 25-32. 
Phase Dynamics and Kinetics with Correlated Temperature Fluctuations L133

[14] H. Haken, Synergetics. An Introduction. Nonequilibrium Phase Transitions and SelfOrganization in Physics, Chemistry, and Biology, third ed., Springer, Berlin (1983).

[15] A. I. Olemskoi, A. V. Khomenko, Three-parameter kinetics of a phase transition, JETP 83 (1996) 1180-1192.

[16] C. W. Gardiner, Handbook of Stochastic Methods, Springer, Berlin (1994).

[17] William H. Press et al., Numerical recipes in $C$ : the art of scientific computing, 2nd ed., Cambridge University Press, New York (1992).

[18] L. D. Landau, E. M. Lifshitz, Statistical Physics, Part 1, 3 rd ed., Pergamon Press, Oxford (1980).

[19] V. L. Popov, Thermodynamics and kinetics of shear-induced melting of a thin layer of lubricant confined between solids, Technical Physics 46 (2001) 605-615.

[20] G. Reiter, A. L. Demirel, J. Peanasky, L. Cai, S. Granick, Physics of Sliding Friction, eds. B. N. J. Persson, E. Tosatti, Kluver, Dordrecht (1995) 119-138.

[21] V. E. Shapiro, Systems near a critical point under multiplicative noise and the concept of effective potential, Phys. Rev. E 48 (1993) 109-120.

[22] A. I. Olemskoi, D. O. Kharchenko, I. A. Knyaz', Phase transitions induced by noise cross-correlations, Phys. Rev. E 71 (2005) 041101-12.

[23] N. G. Van Kampen, Stochastic Processes in Physics and Chemistry, Amsterdam, North-Holland (1981).

[24] J. Zinn-Justin, Quantum Field Theory and Critical Phenomena, Clarendon Press, Oxford (1993).

[25] D. J. Amit, Field Theory, the Renormalization Group, and Critical Phenomena, McGraw-Hill, Inc., New York (1978).

[26] P. Bak, How Nature Works: the Science of Self-Organized Criticality, Springer-Verlag, New York (1996). 